Goldschmidt 2021 Abstract

https://doi.org/10.7185/gold2021.7489

\section{Impact of intraterrestrial microbial life on rock weathering at depth: case studies from cave environments.}

\author{
CELINE PISAPIA ${ }^{1}$, BENEDICTE MENEZ ${ }^{2}$, LAURENT \\ BRUXELLES $^{3}$, MAUD WATKINSON ${ }^{1}$, CEDRIC $^{2}$ \\ CHAMPOLLION $^{4}$, PHILIPPE VERNANT ${ }^{4}$, ERIK \\ DOERFLINGER $^{4}$, HÉLÈNE BOUQUEREL ${ }^{1}$, AUDE \\ ISAMBERT $^{1}$, LÉNA LECOURT ${ }^{2}$ AND EMMANUELLE \\ GÉRARD $^{1}$
}

${ }^{1}$ Université de Paris, Institut de physique du globe de Paris, CNRS UMR 7154, Paris

${ }^{2}$ Université de Paris, Institut de physique du globe de Paris, CNRS UMR 7154

${ }^{3}$ Université Toulouse 2, TRACES, UMR 5608, Toulouse

${ }^{4}$ Université de Montpellier, Geosciences Montpellier, UMR

5243, Montpellier

Presenting Author: pisapia@ipgp.fr

The intraterrestrial biosphere is now considered to be a major component of life on Earth and constitutes up to $70 \%$ of the total biomass of prokaryotes. However, the subsurface as a microbial habitat remains underexplored. It is then mandatory to estimate the impact on the functioning of the Earth system of the activity of this subsurface life, whose metabolisms, mostly decoupled from photosynthesis, should catalyze rock alteration reactions, and consequently influence major current and past biogeochemical cycles, and shape terrestrial landscapes over time.

In this context, cave systems constitute ideal objects to highlight the role of intraterrestrial life on the alteration of the subsurface. They are dynamic geomorphological domains where natural resources (water, nutrients) are stored and where fluidrocks interactions are associated with large rock weathering processes. Moreover, numerous cave systems are described to be formed through ghost-rock processes, an isovolumic alteration process of rocks that leaves in place a soft and highly porous alterite, which can constitute a potential habitat for subsurface microorganisms that could in turn influence mineral weathering processes.

In order to investigate the impact of microbial subsurface life on rock weathering processes, we initiated the geomicrobial study of 2 ghost-rocks karstic systems (South Africa, France). We applied a multi-techniques approach aiming at characterizing (i) the geochemistry and mineralogy of the environment (XRD, Q-ICP-MS, magnetism), (ii) the microbial ecology of the system (metabarcoding) and (iii) (bio)organo-minerals interactions at a micrometric scale in rocks (FTIR and Raman Spectroscopy, SEM-EDX, S-DUV, Tof-SIMS). Alteration mineral phases and notably secondary micrometric (Fe-, $\mathrm{Mn}$-)metallic oxides were identified. They were found closely associated with organic coatings resembling bacterial biofilms, in the microporosity of rocks, and whose biological microbial origin was confirmed after S-DUV and Tof-SIMS analyses. These observations were consistent with metabarcoding results highlighting the presence of uncultivated microorganisms whose metabolisms should be linked to the oxidation/reduction of metallic elements. These observations plead in favour of a potential influence of the activity of microorganisms on rock weathering processes in these environments and confirm the importance of assessing the impact of subsurface life on the functioning of the Earth System. 